\title{
Planejamento Multiobjetivo de Redes Locais sem Fio Utilizando Algoritmos Híbridos
}

\author{
Marlon P. Lima* Ricardo H. C. Takahashi ${ }^{* *}$ \\ Marcos A. M. Vieira ${ }^{* * *}$ Eduardo G. Carrano ${ }^{* * * *}$ \\ * Departamento de Computação e Sistemas, Universidade Federal de \\ Ouro Preto, MG, (e-mail: marlon@ufop.edu.br). \\ ** Departamento de Matemática, Universidade Federal de Minas \\ Gerais, $M G$ (e-mail: taka@mat.ufmg.br) \\ *** Departamento de Ciência da Computação, Universidade Federal de \\ Minas Gerais, MG, (e-mail: mmvieira@dcc.ufmg.br) \\ **** Departamento de Engenharia Elétrica, Universidade Federal de \\ Minas Gerais, MG, (e-mail: egcarrano@ufmg.br)
}

\begin{abstract}
This work presents a novel approach based on hybrid optimization algorithms for planning Wireless Local Area Networks (WLANs). Such an approach is split into two main parts: network structure design and channel assignment enhancement. In the project phase, the quantity, positions, and channels of the access points are planned to take into account their maximum capacities, client demand attendance, and minimum required coverage. Different tests were performed in two scenarios, one using real data from a large-scale WLAN. The results were compared to three approaches and they show that the proposed approach provides solutions that reduce costs and improve the throughput of WLANs.

Resumo: Este trabalho apresenta uma nova abordagem baseada em algoritmos de otimização híbridos para o planejamento de redes locais sem fio (WLANs). Tal abordagem divide-se em duas partes: projeto estrutural da rede e aprimoramento do esquema de atribuição de canais. Na fase de projeto, a quantidade, as posições e os canais dos pontos de acesso são planejados tendo em conta suas capacidades, as demandas de tráfego dos usuários e a cobertura mínima necessária. Diferentes testes foram executados em dois cenários, sendo um deles utilizando dados reais de uma WLAN de grande porte. Os resultados foram comparados com três métodos e demonstram que a abordagem proposta é capaz de fornecer soluções que reduzem os custos e melhoram a vazão das WLANs.
\end{abstract}

Keywords: WLAN; Channel Allocation; Multiobjective Optimization; Genetic Algorithm.

Palavras-chaves: WLAN; Alocação de Canais; Otimização Multiobjetivo; Algoritmos Genéticos.

\section{INTRODUÇÃO}

A crescente busca por mobilidade, combinada à necessidade por redes escaláveis e de baixo custo, justificam o aumento de atenção que as redes locais sem fio (WLAN ou Wireless Local Area Network) têm recebido recentemente. O planejamento das WLANs está muitas vezes focado na abrangência do sinal, com o intuito de reduzir a presença de dead spots (zonas sem cobertura de sinal). Porém, considerações como a localização dos pontos de acesso (APs) e o nível de interferência da rede são frequentemente negligenciadas, apesar de sua importância. Estes fatores tornam-se críticos em aplicações que requerem conexões estáveis e com baixo atraso, como videoconferência, voz sobre IP e multiplataformas de mensagens instantâneas. Essa necessidade vem se tornando ainda maior com a tendência BYOD (Bring Your Own Device) em que os

\footnotetext{
* Os autores agradecem à Universidade Federal de Ouro Preto,
} CAPES, CNPq e FAPEMIG pelo apoio financeiro. usuários demandam redes sem fio de alto desempenho em seus dispositivos móveis praticamente o tempo todo.

A alocação excessiva de APs, além de demandar mais investimento, geralmente leva à degradação do desempenho da rede, devido aos problemas de interferência causados pelo limitado espectro de frequência disponível em WLANs de 2,4 GHz Balbi et al. (2012). Assim, os APs devem estar localizados de modo a garantir a cobertura e o desempenho da rede, além de minimizar a interferência entre eles. Porém, na prática, muitos usuários tendem a reunir-se em uma mesma área Zhao et al. (2016). Tal comportamento, combinado com o aumento da demanda por diferentes serviços, cria uma carga desequilibrada na rede, o que irá comprometer seu desempenho global e a qualidade de serviço oferecida. Portanto, torna-se importante adotar uma política eficiente de associação cliente/AP, com o intuito de maximizar a intensidade de sinal nos clientes mas, ao mesmo tempo, garantir uma distribuição adequada da carga entre os APs. 
Os aspectos citados fazem do planejamento de WLANs um problema de otimização complexo, no qual múltiplos critérios de projeto e restrições técnicas devem ser considerados. A aplicação de métodos exatos para a solução desse problema não é viável, tendo em vista a alta complexidade computacional destes métodos Liu et al. (2015); Zhang et al. (2017). Dessa maneira, meta-heurísticas surgem como alternativas para fornecer soluções razoáveis em tempo computacional aceitável, além de permitirem maior flexibilidade na modelagem. Os Algoritmos Genéti$\cos$ (AGs), por exemplo, têm sido utilizados para a solução de problemas relacionados a WLAN Goudos et al. (2015); Lima et al. (2012). Além disso, os AGs são escaláveis, podendo ser adaptados para lidar com problemas de diferentes dimensões.

Este trabalho propõe uma abordagem baseada em algoritmos híbridos para o planejamento de redes Wi-Fi. Tal abordagem baseia-se em dois algoritmos evolucionários e heurísticas gulosas, e é capaz de fornecer projetos eficientes para WLANs de grande porte, considerando ambientes multi-piso. O método desenvolvido leva em conta o efeito da variação da densidade de usuários nas WLANs. Dois critérios de projeto são considerados: maximização do balanceamento de carga e da relação sinal-ruído. As soluções obtidas atendem à três restrições: cobertura mínima, disponibilidade de canais para operação e largura de banda dos APs. Por fim, um dos cenários utilizados pelo método proposto foi elaborado com dados reais de uma WLAN ESS de grande porte já em funcionamento.

\section{TRABALHOS RELACIONADOS}

Muitos trabalhos existentes para planejamento de WLANs têm focado apenas na cobertura de sinal. Esses estudos não avaliam elementos chave, como a demanda de tráfego requerida, a densidade de usuários, o balanceamento de carga ou a atribuição de canais. Neste sentido, Moreno et al. (2015) propuseram uma técnica para maximizar a cobertura da rede, enquanto a quantidade de APs instalados era minimizada, variando a cobertura dos APs. Esta técnica é conceitualmente similar ao método cell breathing, comumente utilizado em redes de telefonia celular. Os autores modelaram as potências de transmissão dos APs como variáveis, visando reduzir a interferência na rede.

Em um trabalho anterior do próprio autor, Lima et al. (2012) implementou um AG multiobjetivo para planejamento da WLAN que considera a minimização do número de APs e a maximização do balanceamento de carga. A alocação de canais nestes APs foi executada usando um método heurístico. A abordagem foi destinada a redes Wi-Fi outdoor, sem obstáculos. Já Goudos et al. (2015) empregaram um algoritmo de otimização multiobjetivo baseado em biogeografia para projetar uma rede híbrida, 802.11 e LTE (Long Term Evolution). Os objetivos foram maximizar a cobertura da rede e minimizar a potência de transmissão dos APs. Este trabalho não levava em consideração a concentração de clientes nem obstáculos.

Além dos trabalhos já citados, Zhang et al. (2017) propuseram um método baseado em AG para posicionamento de APs e atribuição de canais em uma WLAN. Este algoritmo visa maximizar a cobertura da rede, enquanto a interferência e o número de APs instalados são minimizados. Aspec- tos práticos como obstáculos e aglomeração de usuários são ignorados. Em Liu et al. (2015), os autores implementaram um AG híbrido considerando três objetivos: maximização da cobertura; minimização do número de APs; e da exposição humana aos campos elétricos. O algoritmo foi comparado a uma ferramen-ta de predição da cobertura de WLANs e um algoritmo de Evolução Diferencial em dois cenários. Obstáculos não foram considerados, o que leva à superestimação da cobertura.

Balbi et al. (2012) propuseram um algoritmo centralizado para alocação de canais, baseado no algoritmo DSATUR Brélaz (1979). Este método ajusta suas configurações automaticamente, com base nas condições da rede, considerando a interferência causada por outras WLANs. Já Zhao et al. (2016) utilizaram canais parcialmente sobrepostos para atribuição de frequências em WLANs de superlotadas. Esta abordagem define a quantidade, a posição e o mapeamento de canais para a rede. Tal estratégia baseiase no modelo de sinal-ruído e seu objetivo é maximizar a capacidade geral da rede Wi-Fi. Finalmente, Eisenblatter et al. (2010) desenvolveram um método para lidar com a atribuição de canais em WLANs instaladas em ambientes multipiso. O modelo de otimização multiobjetivo empregado buscou a redução da sobreposição co-canal e a maximização da vazão da rede.

A abordagem empregada neste trabalho difere das demais por agregar em um mesmo estudo diversos pontos importantes no projeto de WLANs: $(i)$ posicionamento de APs, balanceamento de carga e atribuição de canais são tratados de forma conjunta; ( $i$ i) barreiras no ambiente são modelados; (iii) diferentes perfis de clientes são avaliados.

\section{ABORDAGEM PROPOSTA PARA O PLANEJAMENTO DE WLANS}

Foram desenvolvidos dois AGs para o planejamento de WLANs, um para gerar topologias de redes (WLAN NSGA-II) e outro para aprimorar o mapeamento de canais dos APs (Channel Assignment GA). A abordagem recebe como entrada: área de serviço mapeada (obstáculos, limites, etc); conjunto de pontos candidatos (PCs) para instalação dos APs; posições dos clientes; matriz de intensidade de sinal clientes-PCs; consumo de banda estimado e as especificações dos equipamentos.

\subsection{Algoritmo WLAN NSGA-II}

O WLAN NSGA-II foi projetado de forma a lidar com a alocação dos APs e a atribuição dos canais simultaneamente. Este AG multiobjetivo é baseado no NSGA-II Deb et al. (2002) e define a quantidade, o posicionamento e um mapeamento inicial de canais. Para um melhor entendimento do WLAN NSGA-II, serão apresentados primeiro os mecanismos de codificação e decodificação das soluções, e depois seu funcionamento.

\section{Representação e Decodificação das Soluções}

Neste trabalho, foi definido um conjunto discreto de pontos candidatos (PCs) aptos a receber APs que possibilitou o pré-processamento de uma matriz de sinal de cada PC para os clientes, reduzindo consideravelmente o tempo de execução do algoritmo. Com isto, foi utilizada uma 
representação baseada em permutações ${ }^{1}$, que irá garantir a factibilidade das soluções, sem a necessidade de uma alocação excessiva de APs.

O processo de decodificação de uma solução é composto de três etapas. Na Etapa 1, os APs são ativados, um a um, seguindo a ordem definida na permutação, até que as restrições do problema sejam atendidas. Após os requisitos serem cumpridos, as posi-ções restantes da permutação são desconsideradas. Para ilustrar tal conceito, considere a permutação exibida na Figura 1 que, decodificada, resulta na solução da Figura 2a.

Permutação de APs

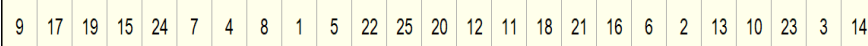

Vetor de Ativaçăa

Figura 1. Representação de uma solução decodificada

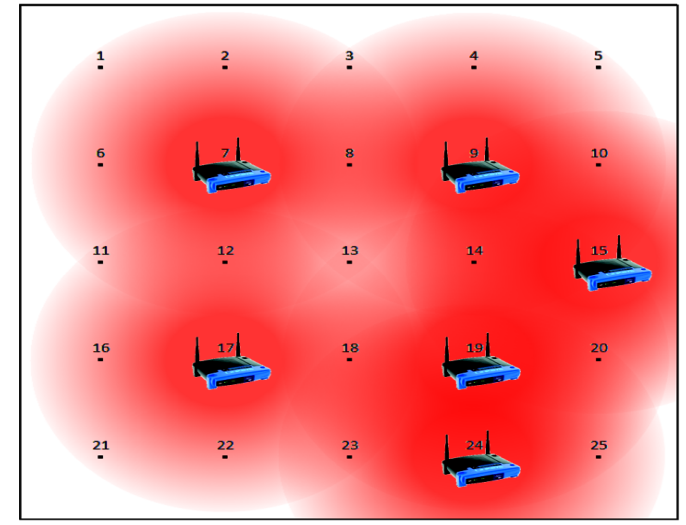

(a) Alocação excessiva de APs

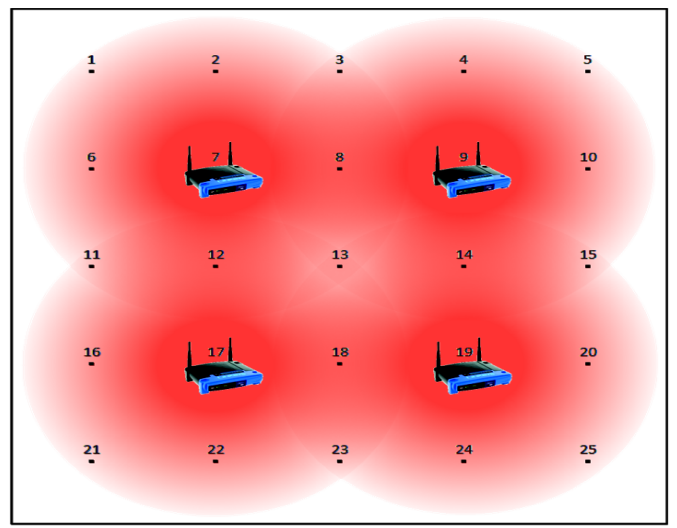

(b) Posicionamento satisfatório dos APs

Figura 2. Disposição de PCs num ambiente sem barreiras

A etapa 2 elimina a instalação de APs desnecessários. Caso a desabilitação do AP (na ordem inversa da ativação) não tenha impacto no atendimento das restrições, então este $\mathrm{AP}$ é desconsiderado; caso contrário, ele permanece ativo. Este processo se repete até que o último AP ativado seja avaliado. A Figura $2 \mathrm{~b}$ exibe a solução após a etapa 2 .

A terceira etapa consiste em associar clientes a APs para a aprimorar o balanceamento de carga. Inicialmente, cada cliente é associado ao AP com maior intensidade de sinal, o que pode conduzir a uma WLAN desbalanceada. Depois, são identificados, para cada cliente, todos os APs alcançáveis que estão operando dentro de sua capacidade,

1 Uma permutação pode ser definida como uma sequência de PCs, em que cada elemento desta sequência aparece somente uma vez. e o AP que minimiza a relação mostrada na equação (1) é escolhido para atender tal cliente.

$$
A P_{j}^{*}=\min _{i}\left[\alpha \cdot \frac{R S S_{\max }-R S S}{R S S_{\max }-R S S_{\min }}+(1-\alpha) \cdot \frac{\operatorname{load}_{A P}}{B W_{A P}}\right]
$$

Nesta expressão, $\alpha=0.7$ é o peso dado à intensidade de sinal (RSS); $R S S_{\min }$ e $R S S_{\max }$ são os limites mínimos e máximos para a RSS; $B W_{A P}$ é a largura de banda do AP.

\section{Redução Dimensional Baseada nas Características Geográficas do Espaço}

Ao se planejar uma WLAN de grande porte, é natural que o número de PCs seja muito alto, o que leva a espaços de busca imensos. Porém, a área de serviço destas redes geralmente abrange várias regiões e, quanto mais afastada elas estão umas das outras, menor será suas interdependências. Considere como exemplo a WLAN de um campus universitário, onde existem dois prédios afastados. A alocação de APs em um prédio tem pouco ou nenhum impacto na escolha dos APs do outro prédio. Logo, qualquer modificação da permutação que promova a troca entre APs desses dois blocos acaba sendo uma operação inútil. Isto também vale para andares afastados no mesmo prédio.

Neste sentido, foi proposta uma representação que proporciona uma redução dimensional do espaço de busca. Ao invés do algoritmo trabalhar com a permutação de todas as PCs simultaneamente, ele quebra essa permutação em subpermutações, tendo como base os subespaços ${ }^{2}$. Porém, a interdependência entre blocos nem sempre é nula. Portanto, é importante definir a ordem em que os blocos serão avaliados. Com isso, cada solução do WLANNSGA-II é composta por duas permutações distintas: $i$ ) permutação dos APs, e; ii) permutação dos blocos. Um exemplo desta representação para uma WLAN com quatro blocos é apresentado na Figura 3, juntamente com o vetor de ativação.

Permutação de APs

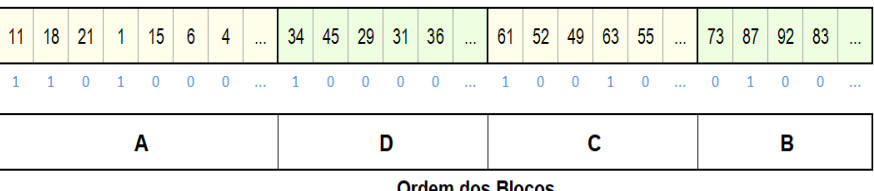

Figura 3. Representação do indivíduo no WLAN NSGA-II

Neste procedimento de decodificação, a permutação dos blocos define a ordem com que eles são avaliados. Seguindo a ordem dos blocos, o primeiro AP não avaliado da subpermutação é ativado e o procedimento passa para o próximo bloco. Ao se alcançar o último bloco da permutação, então o decodificador retorna ao primeiro e refaz o processo. $\mathrm{O}$ processo se repete até que se atinja uma solução que atenda à restrição de cobertura.

\section{Estrutura do Algoritmo}

O WLAN NSGA-II busca maximizar dois objetivos conflitantes: o balanceamento de carga entre os APs e a relação sinal-ruído (SINR) média nos clientes. A primeira operação deste AG consiste em gerar a sequência inicial do blocos. Nesta etapa, verifica-se quantos clientes cada PC é capaz de cobrir, o que permite ranquear os blocos que possuem,

2 Subespaço é uma região da WLAN a ser coberta que sofre pouco impacto das outras regiões da rede. 
em média, PCs que cobrem mais usuários. Feito isto, a população inicial com as permutação das PCs é gerada de forma aleatória. Já o processo de seleção é realizado usando os procedimentos propostos no NSGA-II. Novas permutações dos APs são geradas utilizando o cruzamento PMX e uma versão adaptada do operador Swap Mutation.

O arquivo é criado selecionando as soluções não dominadas da população inicial, e atualizado a cada iteração. Variações da permutação de blocos são avaliadas a cada 25 gerações. É preciso enfatizar que o WLAN NSGA-II opera em duas camadas, ou seja, os operadores que são aplicados às sequências de PCs não são utilizados para as sequências de blocos e vice-versa. Com isso, se a permutação ocorrer na sequência de PCs, assume-se que a ordem dos blocos é fixa para cada indivíduo, e vice-versa. Cada solução possui a relação dos PCs onde serão instalados os APs e o mapeamento de canais a ser utilizado.

Um operador de busca local foi embutido no WLAN NSGA-II para refinamento das soluções, seguindo a estratégia First Improvement, na qual são realizadas buscas até que se encontre uma solução melhor do que a original. Este operador seleciona aleatoriamente 2 APs ativos em cada subpermutação, seguindo a ordem dos blocos do indivíduo. Para cada AP do par, são identificadas 5 PCs vizinhas mais próximas (mensuradas pela RSS). Então, forma-se um conjunto com 25 novas soluções, substituindo os APs escolhidos por seus vizinhos mais próximos. Por fim, este conjunto de soluções é avaliado e, se não for encontrada uma solução que domine a original, o procedimento é repetido para os demais blocos. Este operador de busca local é executado a cada 5 gerações do algoritmo.

O WLAN NSGA-II embarca o conceito de otimização sob múltiplos cenários, uma vez que a mobilidade dos usuários e alterações no consumo de banda dificultam o planejamento das redes 802.11. Tais mudanças podem fazer com que uma solução final seja eficiente para um cenário específico, mas não seja adequada em outros. Assim, o algoritmo desenvolvido considera 10 configurações de rede distintas (variando posição e carga dos clientes), criadas a partir de um perfil de consumo inicialmente estipulado. Neste caso, uma solução precisa ser for factível em todos os 10 cenários em questão.

Por fim, para a atribuição de canais no WLAN NSGA-II foi utilizado uma variação ponderada do algoritmo DSATUR, descrito em detalhes em Lima et al. (2012), que considera a reutilização de canais para APs vizinhos. Este algoritmo é executado antes da avaliação da solução para estimar a SINR. Este método determinístico é rápido e se mostrou adequado para o problema, já que esta operação é realizada milhares de vezes.

\subsection{Algoritmo Channel Assignment GA}

A heurística para atribuição de canais do WLAN NSGAII encontra soluções razoáveis e com tempos de processamento baixo. No entanto, tais soluções podem ser melhoradas com o uso de um algoritmo de otimização eficiente. Em vista disto, foi empregado um AG mono-objetivo para minimizar a interferência nas WLANs, denominado Channel Assignment GA. Este algoritmo foi proposto em Lima et al. (2014) e demonstrou resultados consideravelmente melhores em comparação aos métodos tradicionais. O al- goritmo recebe como entrada uma topologia de WLAN, e fornece como solução um mapeamento de canais mais eficiente em relação à interferências na rede.

O Channel Assignment GA visa maximizar a relação sinal-ruído médio nos clientes. Este algoritmo utiliza uma representação inteira, cruzamento uniforme e mutação de flip. A seleção é realizada usando um torneio binário tradicional com elitismo. Para mais informações sobre este algoritmo, consulte a referência original.

\section{CENÁRIOS DE TESTE E PERFIL DOS USUÁRIOS}

Esta seção descreve os cenários utilizados nos testes da abordagem desenvolvida. Foram selecionados ambientes amplos e distintos, que requerem muitos APs para atender às demandas dos usuários. Diante disso, o cenário 1 representa o primeiro piso de um shopping center, na qual os obstáculos são as paredes no ambiente. Este piso foi escolhido por conter a praça de alimentação do shopping, que apresenta grande concentração de usuários. Os clientes foram criados com uma demanda de tráfego aleatória, variando entre $20 \mathrm{Kbps}$ e $2 \mathrm{Mbps}$, seguindo uma distribuição uniforme. Tal variação contextualiza diversos níveis de atividade dos usuários da rede. A planta baixa deste ambiente foi obtida por meio do Google Maps. Detalhes deste cenário podem ser vistos na Tabela 1.

O cenário 2 foi gerado a partir de dados reais obtidos em estudos realizados na WLAN da Universidade Federal de Ouro Preto - campus Monlevade, que possui uma área total de $30.000 \mathrm{~m}^{2}$, e cinco prédios. A área de serviço desta WLAN, denominada "Minha UFOP WiFi", compreende aproximadamente $14.000 \mathrm{~m}^{2}$. Esta rede conta com $30 \mathrm{APs}$ para atender aproximadamente 1.200 usuários, incluindo alunos e servidores.

$\mathrm{O}$ acesso à Minha UFOP WiFi ocorre em um Captive Portal, que verifica os usuários cadastrados em um servidor de autenticação (FreeRadius). O FreeRadius registra em um banco de dados (Radius Accounting) informações da sessão dos usuários como duração, endereço MAC, quantidade de tráfego de entrada e saída, etc. Estes dados são atualizados ao longo da sessão e armazenados ao fim da mesma, quando é registrado um timestamp. No entanto para uma análise da intensidade de tráfego da rede, foi desenvolvido em um script em Python que coleta informações do Radius Accounting a cada 5 minutos. Este script foi executado durante 1 mês, e após a análise dos dados foi possível traçar um perfil condizente com a quantidade de clientes conectados por AP ao longo do dia, além dos prováveis locais de acesso destes usuários e o consumo de dados na rede.

Foram utilizadas as plantas baixas do campus para identificar as barreiras e tipo de materiais, o que possibilitou definir a atenuação do sinal em diferentes obstáculos. Isto viabilizou o cálculo da matriz de sinal PC para os clientes. Estes níveis de sinal foram validados com medições reais no ambiente. A Tabela 1 exibe um resumo cenário 2.

\section{RESULTADOS COMPUTACIONAIS}

Os resultados obtidos pela abordagem de planejamento proposta são apresentados nesta seção. Os experimentos 
Tabela 1. Dados dos Cenários

\begin{tabular}{r|cc}
\hline Parâmetros & Cenário 1 & Cenário 2 \\
\hline Tamanho da área de serviço & $10.000 \mathrm{~m}^{2}$ & $13.800 \mathrm{~m}^{2}$ \\
Total de pontos candidatos & 2401 & 1061 \\
Número de usuários & 512 & 500 \\
Número de blocos & 1 & 22 \\
Perda penetração em obstáculo & $8 \mathrm{~dB}$ & $3 \mathrm{a} 13 \mathrm{~dB}$ \\
Fator cobertura mínimo & $99 \%$ \\
Ganho de antena dos APs & $5 \mathrm{dBi}$ \\
Potência de transmissão do AP & $100 \mathrm{~mW}$ \\
Limiar mín. recepção de sinal & $-84 \mathrm{dBm}$ \\
Máximo de clientes por AP & 64 \\
Consumo de banda por cliente & $20 \mathrm{Kbps} \mathrm{a} 2 \mathrm{Mbps}$ \\
Capacidade do AP (BW) & $150 \mathrm{Mbps}$ \\
Frequência de operação & $2.4 \mathrm{GHz}$ \\
\hline
\end{tabular}

foram realizados em Matlab R2016a, utilizando um PC Intel i5, 2.5 GHz, 8 GB RAM. Os algoritmos implementados foram executados 33 vezes e configurados com os parâmetros da Tabela 2. As 15 soluções finais obtidas foram submetidas à uma análise de sensibilidade, considerando 1.000 cenários distintos. A escolha dos valores destes parâmetros foi realizada com base em testes preliminares.

Tabela 2. Parâmetros de execução dos algoritmos

\begin{tabular}{r|cc}
\hline Parâmetros & WLAN NSGA-II & Channel Assign. GA \\
\hline Tam. cromossomo & número de PCs & número de APs ativos \\
Número de gerações & $100 / 250$ & 100 \\
Tam. população & 50 & 50 \\
Probab. cruzamento & $80 \%$ (por indiv.) & $40 \%-90 \%$ (por indiv.) \\
Probab. mutação & $10 \%$ (por indiv.) & $3 \%-10 \%$ (por gene) \\
Busca local & a cada 5 gerações & - \\
Permut. dos Blocos & a cada 25 gerações & - \\
\hline
\end{tabular}

Para possibilitar a reprodutibilidade dos resultados, todos os dados de experimentos deste trabalho foram disponibilizados no repositório do $\mathrm{GitHub}^{3}$, contendo: posição e consumo de banda dos clientes; coordenadas dos PCs para instalação de APs; RSS estimada dos clientes para cada PC; posição e atenuação dos obstáculos (em dB); modelo de propagação e sua calibração; parâmetros dos equipamentos; e planta baixa do ambiente.

\subsection{WLAN NSGA-II}

Três métodos distintos para planejamento de redes WiFi e o layout da WLAN de um campus da UFOP foram usados como benchmark para validar as soluções geradas pelo WLAN NSGA-II. O primeiro método foi uma versão modificada do algoritmo K-means, que identifica posições razoáveis para instalação de APs. Nesta versão, a distância Euclidiana foi substituída pela intensidade de sinal. A cada nova iteração, o centróide é redefinido como a posição média dos pontos que pertencem ao seu grupo. O número de centróides (posição dos APs) foi configurado como cada valor inteiro entre as quantidades mínima e máxima de APs empregados nas soluções obtidas pelo WLAN NSGAII. As técnicas de balanceamento de carga e atribuição de canais foram as mesmas utilizadas em todos algoritmos. O intuito deste método é representar um projetista de rede ad hoc.

A segunda abordagem foi a ferramenta comercial para planejamento de WLAN Ekahau Site Survey versão 8.6

\footnotetext{
3 https://github.com/ORCSLab/WLAN_planning/tree/master
}

Ekahau (2016), considerada uma das mais eficientes para planejamento de WLAN e site survey CISCO (2017); MetaGeek (2017). Ela recebe como entradas: um arquivo de mapa, definição de área de serviço (paredes, obstáculos, zona de cobertura), um perfil de consumo esperado e especificações dos equipamentos. Como saída, a ferramenta fornece a quantidade e o posicionamento dos APs e os canais atribuídos. Esta ferramenta comercial foi usada com uma licença de avaliação, gentilmente fornecida pelo representante do software no Brasil.

Os resultados da implementação anterior do WLAN NSGA-II, denominada "Old NSGA-II"Lima et al. (2018) também foram considerados. A diferença entre as versões é que a primeira não lida com ambientes multi-piso, o que permitiu sua aplicação somente ao cenário 1 . Além disto, os operadores genéticos são completamente diferentes dos atuais, uma vez que a versão antiga não trabalha com permutações. Finalmente, a última abordagem foi o atual layout da WLAN do campus universitário do cenário 2.

A Figura 4a exibe resultados de quatro métodos para o primeiro cenário. A nuvem de círculos cinzas representa todas as soluções obtidas nas 33 execuções do WLAN NSGA-II. Após agregar todas soluções, foi possível obter o conjunto final da aproximação da fronteira Pareto, representadas por triângulos azuis. Os círculos vermelhos são as soluções obtidas pelo Old NSGA-II. Os quadrados verdes são as soluções fornecidas pelo Ekahau e as estrelas magenta são os resultados do algoritmo k-means.

Para o algoritmo K-means foram geradas soluções empregando o mesmo número de APs encontrados pelo WLAN NSGA-II (entre 12 e 16 APs). Porém, se comparado aos demais, este método não produziu bons resultados. No caso do Ekahau, a variação de parâmetros como potência de transmissão do AP e intensidade mínima de sinal recebido geralmente resultam em layouts de rede distintos. Assim, suas soluções foram razoáveis em termos de SINR, mas ruins com relação ao balanceamento da rede. Isto se deve ao fato de tal ferramenta não levar em consideração as aglomerações dos usuários.

Apesar dos resultados obtidos pelo Old NSGA-II superarem o Ekahau e o K-means, a maior parte de suas soluções foram piores do que o WLAN NSGA-II. Apenas as soluções do Old NSGA-II que oferecem alto SINR foram superiores às do algoritmo proposto. Tais resultados são bastante significativos, uma vez que este último algoritmo é capaz de lidar com cenários mais complexos e também com ambientes multi-piso. Por fim, as soluções do WLAN NSGA-II estão bem espalhadas ao longo da fronteira.

A Figura 4b exibe os resultados para o segundo cenário. Todas as soluções não-dominadas obtidas pelo WLAN NSGA-II foram superiores às outras abordagens utilizadas como benchmark. Além disto, as soluções das 33 execuções estão bem próximas umas das outras e espalhadas ao longo da aproximação da fronteira Pareto. No caso do Ekahau, as soluções obtidas também foram razoáveis em termos de relação sinal-ruído, mas ruins em balanceamento.

No que diz respeito à configuração atual da UFOP, seu desempenho foi muito ruim. Isto se deve ao fato dos administradores da rede, na época de seu planejamento, levarem em consideração apenas sua cobertura. Com isso, 


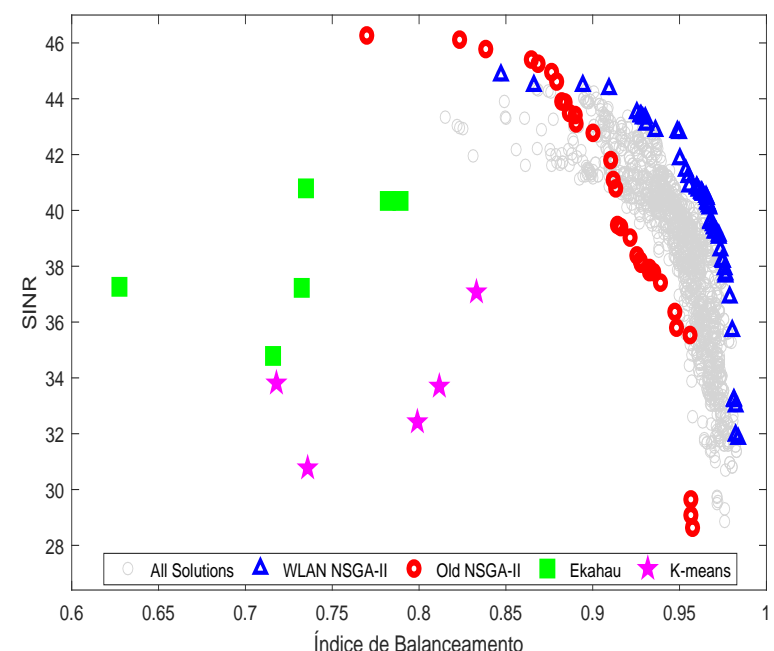

(a) Cenário 1

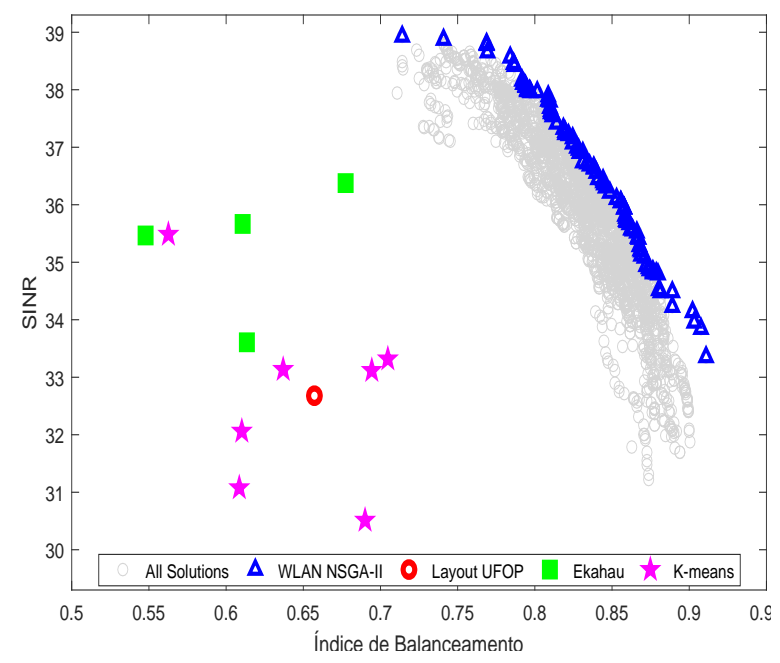

(b) Cenário 2

Figura 4. Teste comparativo entre as abordagens

a demanda dos usuários foi deixada de lado, já que não foi realizado um estudo sobre o perfil dos usuários ou sua localização, como o que foi desenvolvido neste artigo. Até mesmo pela experiência como usuário, percebe-se que a rede atual não é adequada.

O tempo computacional gasto pelo WLAN NSGA-II nos cenários 1 e 2 foi de aproximadamente 4 e 8 horas para cada execução, respectivamente. Este tempo total de execução pode ser reduzido por meio de uma paralelização simples de cada uma das execuções, uma vez que o ganho é linear. Considerando que este é um problema de planejamento, tais valores se mostraram razoáveis, uma vez que a solução fornecida pelo algoritmo poderá ser utilizada durante alguns anos. Vale a pena destacar que a abordagem desenvolvida é escalável, o que possibilita sua aplicação com eficiência em WLANs de pequenos ou grande porte.

\subsection{Channel Assignment GA}

O Channel Assignment GA foi executado 33 vezes, para cada uma das soluções não-dominadas obtida nas execuções do WLAN NSGA-II. Consequentemente, depois desta etapa surgiram algumas soluções dominadas. Então foi realizada um filtragem por não-dominância, e o conjunto final foi truncado em 15 soluções, baseado na crowding distance. A Figura 5a exibe os resultados deste algoritmo para o cenário 1. Este gráfico mostra também o percentual de usuários sofrendo interferência na rede. De modo geral, o DSATUR obteve resultados satisfatórios e muito próximos do Channel Assignment GA quando o nível de interferência da rede é moderado. No entanto, quando a interferência no ambiente aumenta, o AG oferece melhor SINR. É preciso deixar claro que a RSS cliente-AP é sempre a mesma, ou seja, não é considerado nenhuma reassociação cliente-AP. Neste caso, o que muda é apenas o mapeamento de canais fornecido pelo algoritmo.

Quanto ao cenário 2 (Figura 5b), no qual são empregados entre 24 a 30 APs, é possível verificar que o Channel Assignment GA supera com folga o DSATUR, devido a maior complexidade do cenário. Note que esta diferença média observada de $3 \mathrm{~dB}$ entre os dois métodos é significativa em termos de qualidade de sinal da rede. Vale destacar que a melhor solução obtida pelos 2 algoritmos é adotada para o mapeamento de canais. Finalmente, para cada solução não-dominada obtida pelo WLAN NSGA-II, o Channel

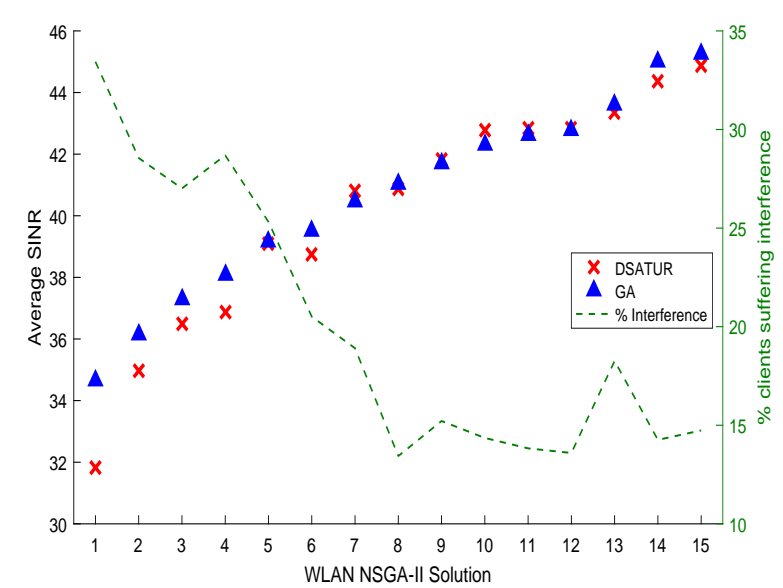

(a) Cenário 1

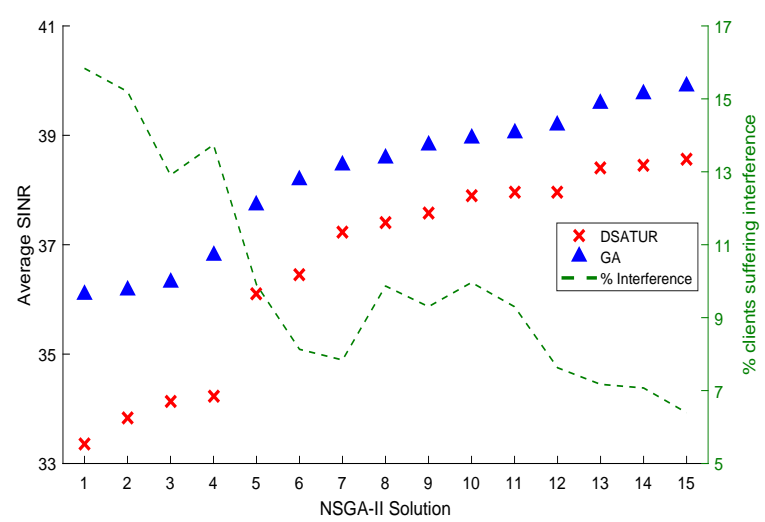

(b) Cenário 2

Figura 5. Teste - Channel Assignment GA x DSATUR

\subsection{Análise de Sensibilidade}

As soluções obtidas pela abordagem proposta foram avaliadas em 1.000 configurações de clientes distintas (variando posição e demanda). Assim, os intervalos de confiança de 95\% dos critérios de cobertura, índice de balanceamento e 
SINR para o Cenário 1 foram avaliados e foi possível perceber que a alteração no perfil dos clientes não ocasionou redução significativa da qualidade na maioria das soluções. O fato de não haver queda considerável em nenhum dos critérios do problema sugere que a adoção da estratégia de múltiplos cenários surtiu um efeito positivo.

No caso do cenário 2, após a análise de sensibilidade foi possível perceber que algumas soluções que apresentam índice de balanceamento ruim ou fornecem SINR baixa. Este aspecto justifica mais uma vez a adoção de uma abordagem multiobjetivo para o planejamento de WLANs, uma vez que é possível selecionar designs de rede que sejam equilibrados em ambos objetivos.

Com relação à análise de falhas, foram avaliadas perdas aleatórias em 1, 2 ou 3 APs. Embora a SINR e o balanceamento de carga também sejam afetados por problemas em APs, somente a cobertura da rede foi considerada, pois entende-se que estas são condições temporárias e críticas. Assim, para o primeiro cenário, observou-se que cinco soluções têm baixa probabilidade de atender ao requisito de $99 \%$ de cobertura, o que indica que elas podem ser ignoradas como candidatas à implementação. Já para o cenário 2 , três soluções têm menor probabilidade de oferecer cobertura ampla na rede, em caso de falhas de APs. Além disto, tais soluções oferecem baixa SINR para os clientes da WLAN.

Esta análise de sensibilidade, em conjunto com as soluções que foram avaliadas, são o resultado final da abordagem de planejamento de WLAN proposta. Tal análise pode fornecer informações valiosas para a seleção final do design de redes locais sem fio.

\subsection{Soluções Sugeridas}

A solução sugerida para o Cenário 1 é exibida na Figura 6. As cores vermelho, verde e azul correspondem aos canais 1,6 e 11, respectivamente. É visível que o algoritmo distribuiu os APs muito bem entre os três clusters, sem sobrecarregar nenhum dispositivo. Até mesmo com um pequeno número de APs instalados (14), foi possível atender a todos os requisitos do problema. Sob o ponto de vista dos autores, esta solução é uma opção razoável a ser adotada, porque ela representa um equilíbrio aceitável entre os objetivos e cobertura razoável (mais de $98 \%$ ), mesmo para o caso de falha em um dos seus APs.

Para o segundo cenário, uma análise criteriosa das soluções propostas mostra que a abordagem desenvolvida conseguiu fornecer boas alternativas de projeto. Assim como no primeiro cenário, a heurística implementada distribuiu os APs de forma apropriada e alocou canais de modo que a interferência causada fosse moderada. A Figura 7 exibe uma opção de projeto selecionada, que se mostrou apta a atender às demandas dos clientes com níveis de cobertura aceitáveis. Esta solução parece oferecer o melhor compromisso entre os três objetivos e obteve bons resultados no critério falha dos APs. Perceba também que a quantidade de pontos pretos (clientes não atendidos) é muito baixa. Por fim, os pontos coloridos, mas que não estão visivelmente ligados aos APs, estão sendo atendidos por pontos de acesso localizados em outros andares.

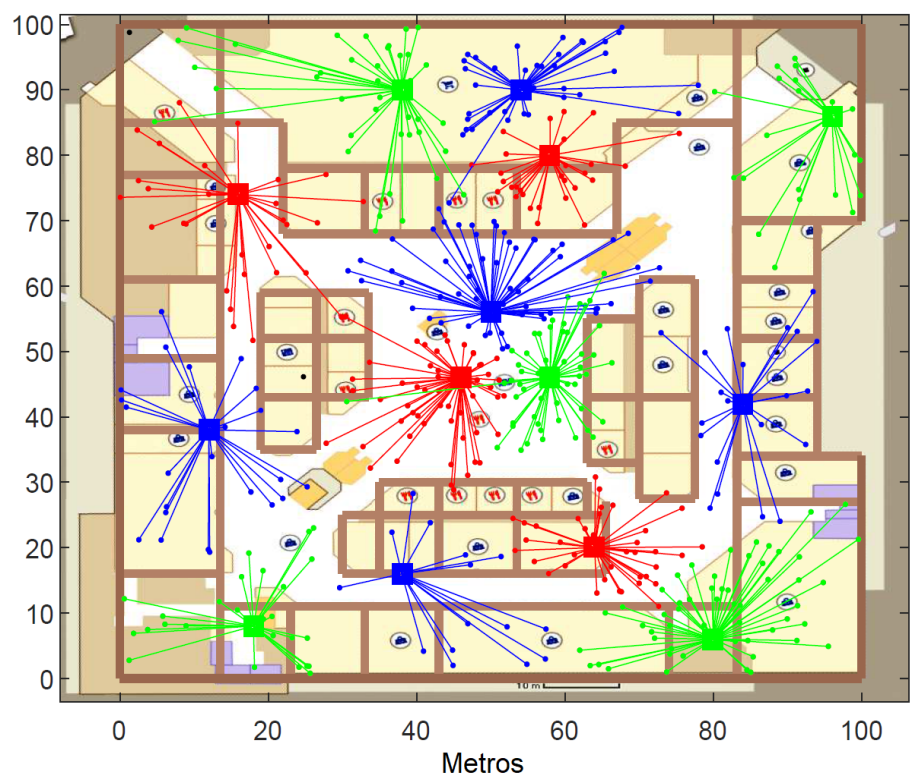

Figura 6. Cenário 1 - Solução 9

É preciso salientar que o cenário 2 é muito mais complexo que o anterior, uma vez que seu espaço de busca é maior, a área de serviço da rede abrange vários pisos e existem muitos obstáculos no ambiente. Além disso, apesar da maior parte dos clientes estarem espalhados no campus, existe uma concentração de usuários em uma área específica, que corresponde ao Diretório Acadêmico, refeitório e cantina. Finalmente, boa parte destes clientes estão localizados em uma área aberta, onde a instalação de APs não é viável.

\section{CONCLUSÃO}

Nos últimos anos, grandes projetos de redes locais sem fio têm sido implantados em ambientes como universidades, shoppings centers e aeroportos. A popularização desta tecnologia trouxe consigo um aumento no desempenho e cobertura, além de possibilitar o desenvolvimento de novas aplicações. No entanto, a adoção em massa das WLANs resultou em cenários com elevada densidade de usuários, gerando problemas de desequilíbrio e interferência na rede. Neste trabalho foi desenvolvida uma abordagem para planejamento de WLANs que sugere a quantidade e o posicionamento de APs no espaço, além de propor um esquema eficiente de alocação de canais. Tal abordagem é baseada em um AG multiobjetivo, que aloca os APs e fornece um mapeamento inicial de canais simultaneamente, e também por um AG mono-objetivo, empregado para aprimorar a atribuição de canais. Os AGs e heurísticas propostos embarcam no processo de otimização conhecimento específico sobre o problema tratado, o que aumenta sua eficiência.

A abordagem de planejamento proposta foi avaliada em dois cenários distintos, levando em conta necessidades diferentes para os usuários. Foram obtidas soluções interessantes sob o ponto de vista do trade-off entre equilíbrio de carga e intensidade de sinal. Na comparação com outras metodologias, a abordagem proposta obteve resultados consideravelmente superiores. Por fim, as soluções obtidas pela foram submetidas a uma análise de sensibilidade, com relação a alterações no perfil dos usuários e falhas nos APs. Tal análise se mostrou útil para o decisor na escolha da melhor configuração de rede. 


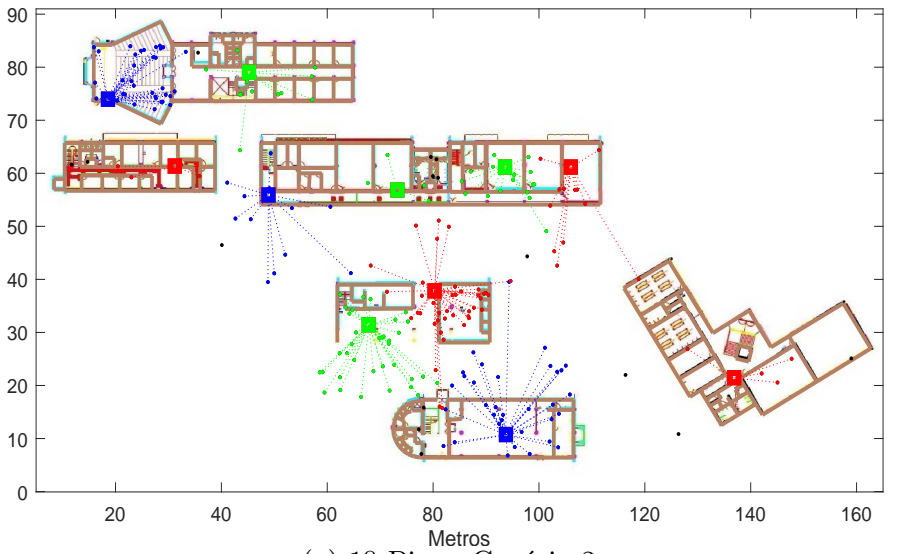

(a) $1^{\circ}$ Piso - Cenário 2

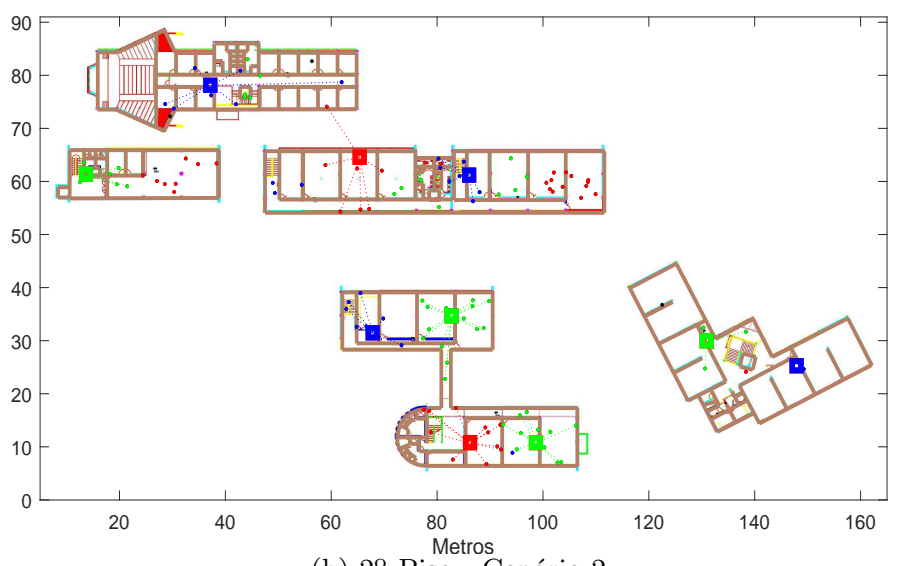

(b) $2^{\circ}$ Piso - Cenário 2

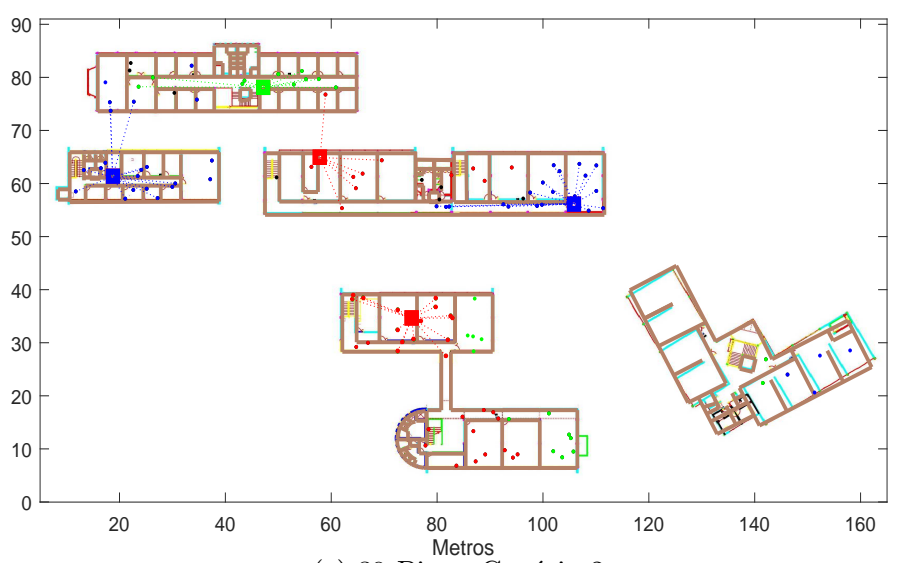

(c) $3^{\circ}$ Piso - Cenário 2

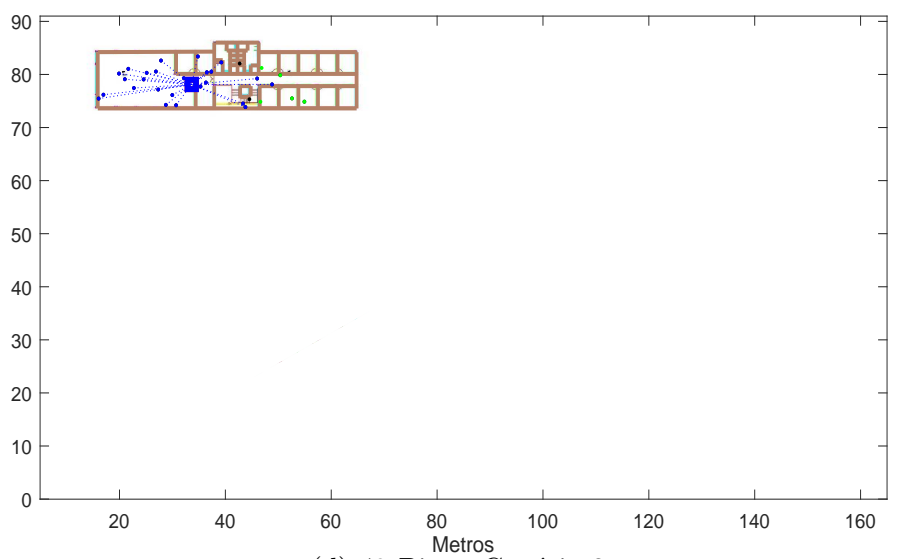

(d) $4^{\circ}$ Piso - Cenário 2

Figura 7. Solução sugerida para a WLAN do Campus

\section{REFERÊNCIAS}

Balbi, H., Fernandes, N., Souza, F., Carrano, R., Muchaluat-Saade, D., and Magalhães, L. (2012). Centralized channel allocation algorithm for IEEE 802.11 networks. In Global Information Infrastructure and Networking Symposium (GIIS), 1-7.

Brélaz, D. (1979). New methods to color the vertices of a graph. Commun. ACM, 22(4), 251-256.

CISCO (2017). Site survey tools. https:// learningnetwork. cisco.com/thread/24648

(Accessed: May 18, 2017).

Deb, K., Pratap, A., Agarwal, S., and Meyarivan, T. (2002). A fast and elitist multiobjective genetic algorithm: NSGA-II. IEEE Transactions on Evolutionary Computation, 6(2), 181-197.

Eisenblatter, A., Gross, J., Punal, O., and Schweiger, J. (2010). A two-stage approach to WLAN planning: Performance evaluation along the Pareto frontier. In Modeling and Optimization in Mobile, Ad Hoc and Wireless Networks (WiOpt), 227-236.

Ekahau (2016). Ekahau Site Survey, version 8.6 [computer software]. Reston, VA, USA. URL http://www . ekahau. com/wifidesign/ekahau-site-survey.

Goudos, S.K., Plets, D., Liu, N., Martens, L., and Joseph, W. (2015). A multi-objective approach to indoor wireless heterogeneous networks planning based on biogeography-based optimization. Computer Networks, 91, 564-576.

Lima, M.P., Carrano, E.G., and Takahashi, R.H.C. (2012). Multiobjective planning of wireless local area networks (WLAN) using genetic algorithms. In IEEE Congress on Evolutionary Computation, 1-8.

Lima, M.P., Rodrigues, T.B., Alexandre, R.F., Takahashi, R.H.C., and Carrano, E.G. (2014). Using evolutionary algorithms for channel assignment in 802.11 networks. In Computational Intelligence for Communication Systems and Networks (CIComms), 1-8.

Lima, M.P., Takahashi, R.H.C., Vieira, M.A.M., and Carrano, E.G. (2018). Hybrid multicriteria algorithms applied to structural design of wireless local area networks. Applied Intelligence. doi:10.1007/s10489-018-1165-7.

Liu, N., Plets, D., Goudos, S.K., Martens, L., and Joseph, W. (2015). Multi-objective Network Planning Optimization Algorithm: Human Exposure, Power Consumption, Cost, and Capacity. Wirel. Netw., 21(3), 841-857.

MetaGeek (2017). Top Wireless WiFi Site Survey Software. http://forums.metageek.com/ (Accessed: May 18, 2017).

Moreno, J., Domingo, M., Valle, L., Lopez, J.R., Torres, R.P., and Basterrechea, J. (2015). Design of Indoor WLANs: Combination of a ray-tracing tool with the BPSO method. IEEE Antennas and Propagation Magazine, 57(6), 22-33.

Zhang, Z., Tian, J., and Zhu, Z. (2017). A multi-objective WLAN planning method. In 2017 International Conference on Information Networking (ICOIN), 86-91.

Zhao, W., Nishiyama, H., Kato, N., and Hamaguchi, K. (2016). DAPA: Optimization in Wireless Networks Through a Combined Design of Density of Access Points and Channel Allocation. IEEE Transactions on Vehicular Technology, 65(5), 3715-3722. 